\title{
Dados abertos para o fomento da transparência e inovação: o Caso da UFRN
}

\section{Title: Open data for promotion of transparency and innovation: the case of UFRN}

\author{
Artigo submetido à Edição Especial sobre Transparência em Sistemas de \\ Informação \\ Gibeon Soares de Aquino Júnior ${ }^{1}$, Elias Jacob de Menezes Neto', Gusttavo \\ Henrique Nascimento Sousa Silva ${ }^{1}$, Jean Guerethes ${ }^{1}$, Renan de Oliveira Silva ${ }^{2}$ \\ Universidade Federal do Rio Grande do Norte (UFRN) \\ Natal, RN - Brasil \\ ${ }^{2}$ Instituto Federal de Educação, Ciência e Tecnologia do Rio Grande do Norte (IFRN) \\ João Câmara, RN - Brasil

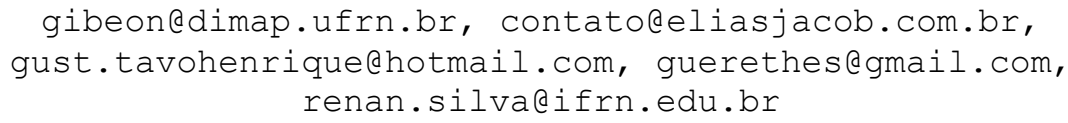

\begin{abstract}
The recent rise of open data movements in Brazil is noticeable. It was allowed mainly by advances in legislation on transparency and integrity in the Public Sector. The adoption of open data by public institutions, aligned with a policy of support and encouragement to the consumption of them, provides innumerable benefits to society and the institution itself. In this sense, this article aims at presenting how UFRN's adopted strategy contributed to promoting satisfactory transparency in public administration while has fostered an ecosystem of innovation.
\end{abstract}

Keywords. Transparency; Innovation; Open Data; ICT.

Resumo. É notável a ascensão recente dos movimentos de dados abertos no Brasil, viabilizados principalmente pelos avanços na legislação sobre transparência e integridade no Setor Público. A adoção de dados abertos por parte de instituições públicas, alinhada com uma política de suporte e fomento ao consumo desses dados possibilita inúmeros benefícios para a sociedade e a própria instituição. Neste sentido, o objetivo deste artigo é apresentar como a estratégia de abertura de dados adotada pela UFRN contribuiu para promover a transparência na administração pública, ao mesmo tempo que fomentou um ecossistema de inovação.

Palavras-Chave. Transparência; Inovação; Dados Abertos; TIC. 


\section{Introdução}

É notável a ascensão recente dos movimentos de dados abertos no Brasil. Esta mobilização tem sido incentivada pelos avanços na legislação sobre transparência e integridade no Setor Público, mas também impulsionada principalmente pelas recentes divulgações de corrupção sistêmica. Apesar deste recente alvoroço em torno desta filosofia, o conceito e os seus princípios norteadores não são novos. Eles já vêm sendo trabalhados e amadurecidos há bastante tempo em diversos países, como Estados Unidos, Reino Unido e Canadá. Os princípios fundamentais que embasam a filosofia de dados abertos, particularmente os relacionados à transparência e ao 'direito de saber' do cidadão, têm sido considerados por governos desde o século XVIII. Particularmente, nos Estados Unidos estes são tratados formalmente desde a década de 50 (PARKS, 1957).

Vários países vêm firmando acordos com o objetivo de promover e fortalecer a implementação dos princípios relacionados à transparência em governos. Um exemplo é a OGP ${ }^{\prime}$ (Open Government Partnership), que é uma iniciativa internacional com a missão de difundir e fomentar práticas governamentais para a transparência dos governos, acesso à informação pública e participação social. Esta iniciativa foi lançada em 20 de setembro de 2011 e tem como um dos membros fundadores o Brasil. Apesar do Brasil ter se engajado fortemente nas ações de dados abertos apenas na última década, incentivado principalmente pelos compromissos assumidos com a OGP, os resultados recentes demonstram que a estratégia adotada foi bem adequada. Particularmente, o Brasil atingiu o oitavo lugar no ranking mundial de Dados Abertos da OKFN $^{2}$ (Open Knowledge Foundation) de 2017, conquistando a liderança na América Latina (GOVERNO DO BRASIL, 2018). Um dos fatores de sucesso está em como a estratégia de abertura de dados está alinhada e sustentada pelas Tecnologias de Informação e Comunicação (TICs).

De fato, o advento das TICs tem um papel predominante no avanço das políticas de transparência pública e empoderamento do cidadão (VIEIRA e SANTOS, 2010). As TICs são fortes aliadas às estratégias de combate à corrupção (ANDERSEN, 2009). Mais ainda, as TICs podem e devem ser utilizadas para a criação de uma cultura de transparência, e que a popularização do governo aberto e governo eletrônico, aliado às mídias sociais, podem ser utilizadas pela sociedade como ferramentas efetivas contra a corrupção (BERTOT et al., 2010; PICAZO-VELA et al. 2013).

Uma outra perspectiva complementar é que os dados abertos são capazes de promover um espaço para desenvolvimento de inovações (CHAN, 2013; ZUIDERWIJK et al, 2014). As entidades públicas geram grandes quantidades de dados, de interesse da sociedade, que podem ser combinados e transformados, produzindo potencialmente informações e ferramentas de maior valor. A publicação desses dados, com devido suporte e fomento ao consumo pelo cidadão, pode propiciar mais controle social, transparência, cidadania ativa, cooperação, melhorias na administração pública, bem como a criação de novas ferramentas e produtos para o bem-estar social (KUCERA, 2015).

\footnotetext{
' https://www.opengovpartnership.org/

${ }^{2}$ https://index.okfn.org/
}

iSys: Revista Brasileira de Sistemas de Informação (isys: Brazilian Journal of Information Systems) http://seer.unirio.br/index.php/isys/ 
Neste sentido, o objetivo deste artigo é apresentar como a estratégia de abertura de dados adotada pela UFRN contribuiu simultaneamente para promover a transparência na administração pública, ao mesmo tempo que fomentou um ecossistema de inovação. Particularmente, a iniciativa viabilizou o desenvolvimento de várias ferramentas e análise de dados de interesse da comunidade acadêmica. $O$ mais surpreendente foi que estes desenvolvimentos foram realizados de forma completamente descentralizada e sem necessidade de qualquer indução por parte da gestão. Acreditamos que tais resultados não foram fruto do mero acaso, e sim das direções adotadas ao longo da implantação. O mais interessante é que as condições necessárias para tais resultados podem ser reproduzidas em outras instituições públicas, desde que haja o devido interesse e engajamento.

Esse artigo está organizado da seguinte maneira: na Seção 2 é apresentada a fundamentação teórica relacionada a dados abertos na administração pública. Na Seção 3, a estratégia adotada pela UFRN é apresentada em detalhes, com os princípios e práticas utilizados. Na Seção 4, os resultados obtidos são apresentados e discutidos. Finalmente, na Seção 5 apresentam-se as oportunidades e considerações finais.

\section{Dados Abertos na Administração Pública}

Pode-se definir a democracia de diferentes formas, mas não existe definição que possa deixar de incluir em seus conotativos a visibilidade ou transparência do poder. A principal proposta dos primeiros regimes democráticos foi desvelar o núcleo duro e oculto do poder exercido por pequenos grupos. Essa foi, inclusive, uma das razões da sua superioridade em relação ao Estado absoluto, em que eram defendidos os poderes ocultos - arcana imperii. O segredo fazia parte da prática política relativa ao Estado moderno, sendo sua presença inversamente proporcional ao grau de liberdade em determinado governo: tanto mais livres eram os súditos quanto mais acessíveis fossem os fundamentos de todos os atos de poder (BOBBIO, 2015).

No contexto brasileiro atual, existe uma ampliação da cobrança da sociedade por maior transparência na Administração Pública. A ampla divulgação pela mídia de um quadro de corrupção sistêmica na cúpula dos diversos poderes requer uma mudança de paradigma na forma de administrar a coisa pública, e, para tanto, mostra-se urgente a criação e a manutenção de novas práticas de gestão, transparência e participação social.

A importância dessa temática pode ser comprovada pela recente proliferação de legislação sobre transparência e integridade no Setor Público, como é o caso das Leis 12.527/11 e 12.846/13, Decretos 7.724/12, 8.420/15 e 8.777/16, Portarias CGU 909/15 e 784/16 e Instrução Normativa Conjunta MPOG/CGU nº 01/2016. Além disso, há um Projeto de Lei (PL7.804/2014), em tramitação na Câmara dos Deputados, que trata sobre a disponibilização de dados em formato aberto por todos os poderes (Executivo, Legislativo e Judiciário) em todas as esferas (federal, estadual e municipal).

Ao invés de um retorno a uma potencial democracia direta associada às novas TICs, os dados abertos governamentais são um modelo viável de aumento da participação democrática proporcionada pela tecnologia. A Open Definition define o significado do adjetivo "aberto" como delimitadora de algo que pertence à categoria de bem comum, com participação livre e interoperabilidade maximizada. Segundo a versão 2.1 daquela definição, "o conhecimento é aberto se qualquer pessoa está livre para acessá-lo, utilizá-lo, modificá-lo, e compartilhá-lo - restrito, no máximo, a medidas 
que preservam a proveniência e abertura." (OPEN KNOWLEDGE FOUNDATION, 2018). A partir dessa definição, o Poder Executivo federal estabeleceu que são abertos os "dados acessíveis ao público, representados em meio digital, estruturados em formato aberto, processáveis por máquina, referenciados na internet e disponibilizados sob licença aberta que permita sua livre utilização, consumo ou cruzamento, limitando-se a creditar a autoria ou a fonte" (BRASIL, 2016).

De acordo com o Portal Brasileiro de Dados Abertos (2018), mantido pelo Poder Executivo Federal, os dados abertos são regidos por oito "princípios" essenciais, de modo que devem ser:

- Completos. Todos os dados públicos são disponibilizados. Dados são informações eletronicamente gravadas, incluindo mas não se limitando a documentos, bancos de dados, transcrições e gravações audiovisuais. Dados públicos são dados que não estão sujeitos a limitações válidas de privacidade, segurança ou controle de acesso, reguladas por estatutos.

- Primários. Os dados são publicados na forma coletada na fonte, com a mais fina granularidade possível, e não de forma agregada ou transformada.

- Atuais. Os dados são disponibilizados o quão rapidamente seja necessário para preservar o seu valor.

- Acessíveis. Os dados são disponibilizados para o público mais amplo possível e para os propósitos mais variados possíveis.

- Processáveis por máquina. Os dados são razoavelmente estruturados para possibilitar o seu processamento automatizado.

- Acesso não discriminatório. Os dados estão disponíveis a todos, sem que seja necessária identificação ou registro.

- Formatos não proprietários. Os dados estão disponíveis em um formato sobre o qual nenhum ente tenha controle exclusivo.

- Livres de licenças. Os dados não estão sujeitos a regulações de direitos autorais, marcas, patentes ou segredo industrial. Restrições razoáveis de privacidade, segurança e controle de acesso podem ser permitidas na forma regulada por estatutos.

As novas possibilidades de uso de dados abertos para melhoria da transparência, participação e eficiência das políticas públicas demonstram que um dos grandes desafios do Brasil, no século XXI, será a melhoria da governança no setor público, ou seja, o desenvolvimento de "[...] um conjunto de mecanismos de liderança, estratégia e controle postos em prática para avaliar, direcionar e monitorar a gestão, com vistas à condução de políticas públicas e à prestação de serviços de interesse da sociedade" (TRIBUNAL DE CONTAS DA UNIÃO, 2014, p. 5-6).

No mesmo sentido, o art. $2^{\circ}$, inciso IX da Instrução Normativa Conjunta MPOG/CGU n ${ }^{\circ}$ 01/2016 especifica que governança no setor público “compreende essencialmente os mecanismos de liderança, estratégia e controle postos em prática para avaliar, direcionar e monitorar a atuação da gestão, com vistas à condução de políticas públicas e à prestação de serviços de interesse da sociedade”.

Diferentemente da governança corporativa, muito difundida em grandes empresas, a governança no setor público apresenta desafios próprios, seja em virtude do tamanho do Estado necessário para viabilizar a concretização dos direitos humanos 
previstos na Constituição Federal de 1988, seja porque o setor público possui responsabilidades de transparência e accountability ${ }^{3}$ muito maiores que o setor privado.

O sistema de governança no setor público é composto por um complexo cenário, no qual diversos atores sociais movimentam-se com a finalidade de obter a boa gestão da coisa pública. Dessa forma, envolve:

\begin{abstract}
Estruturas administrativas (instâncias), os processos de trabalho, os instrumentos (ferramentas, documentos etc.), o fluxo de informações e o comportamento de pessoas envolvidas direta, ou indiretamente, na avaliação, no direcionamento e no monitoramento da organização (TRIBUNAL DE CONTAS DA UNIÃO, 2014, p. 28).
\end{abstract}

É através da governança no setor público que o Estado poderá maximizar o uso dos recursos disponíveis para viabilizar o seu projeto de proteção da democracia e de transformação social. É possível afirmar, portanto, que o objetivo da governança no setor público é garantir a consecução do interesse público em todos os momentos. Para isto, exige-se a observação de uma série de requisitos, dentre eles a garantia de transparência e a definição das intervenções necessárias para otimizar o alcance dos resultados esperados (INTERNATIONAL FEDERATION OF ACCOUNTANTS, 2014, p. 10).

Diante desse cenário, está em prática uma estratégia de governança digital segundo a qual o Estado brasileiro deve utilizar as TICs para oferecer seus serviços de forma mais eficiente. Além disso, um dos principais objetivos dessa estratégia é a participação da sociedade no processo democrático tanto na tomada de decisões, quanto em relação à participação na responsabilidade, transparência e efetividade das ações do governo. A ideia de governança digital está, portanto, intimamente ligada aos processos de democracia monitória, deliberativa e participativa.

Ruijer, Grimmelikhuijsen e Meijer (2017) caracterizam a democracia deliberativa como o processo que enfatiza o debate aberto para a tomada de decisões coletivas, sendo estas decisões formadas a partir da opinião pública e da vontade geral, valorizando os diferentes pontos de vista construídos a partir das informações disponíveis. Logo, essa estratégia abrange a ideia de democracia deliberativa, na medida em que incentiva a colaboração da sociedade no processo de tomada de decisões.

Igualmente, visualiza-se a manifestação da democracia participativa na política de dados abertos através da ideia de que os cidadãos colaboram diretamente com o governo, deixando o povo de ser mero usuário de serviços públicos digitais, tornando-se agentes ativos no processo de formação, implementação e monitoramento das atividades administrativas.

Dentro do cenário democrático, os benefícios da abertura de dados governamentais são bastante diversos. Podem-se beneficiar dessa política tanto o Governo quanto a sociedade civil (especialmente empresários, pesquisadores e o próprio setor privado), que podem explorar, inclusive economicamente, as informações obtidas.

\footnotetext{
${ }^{3}$ Nos termos do art. $2^{\circ}$, inciso I da Instrução Normativa Conjunta MPOG/CGU n ${ }^{\circ}$ 01/2016, accountability significa o "conjunto de procedimentos adotados pelas organizações públicas e pelos indivíduos que as integram que evidenciam sua responsabilidade por decisões tomadas e ações implementadas, incluindo a salvaguarda de recursos públicos, a imparcialidade e o desempenho das organizações"
} 
Um dos benefícios da abertura de dados é a promoção da transparência e da possibilidade de controle da coisa pública pelo cidadão. A transparência, associada ao princípio constitucional da publicidade e ao direito fundamental de acesso à informação, permite à sociedade controlar o uso dos bens públicos, o que inclui os recursos destinados aos mais diversos setores. A partir do momento em que o cidadão tem acesso a uma base de dados digital e atualizada sobre os atos praticados pela Administração Pública, ele tem a possibilidade de tornar-se mais vigilante e capaz de identificar ações corruptas e ímprobas.

Além disso, o viés econômico é extremamente importante do ponto de vista da Administração Pública, uma vez que a abertura de dados tem o condão de gerar maior eficiência no uso dos recursos públicos. Nesse sentido, o European Data Portal (2018) esclarece: "A publicação de dados abertos permite o compartilhamento de informações dentro de governos em formatos interoperáveis legíveis por máquina, o que resulta na redução de custos de troca de informações e integração de dados, gerenciamento de fluxos de dados, redução de erros por ter uma cópia em vez de múltiplas, etc. Isto resulta em melhor gerenciamento de dados, em termos de qualidade e eficiência, bem como uma redução geral nos custos administrativos. Na verdade, a grande área de Manchester estimou que as solicitações de liberdade de informação custam aos órgãos públicos mais de $£ 4$ milhões por ano, enquanto mais de 600 funcionários públicos por dia não conseguem encontrar ou usar os dados necessários para seus trabalhos, custando às autoridades mais de $£ 8.5$ milhões por ano. Ao quebrar os silos que existem entre os vários departamentos, órgãos e camadas de governo e permitindo um fluxo de dados fluido pode ter ganhos substanciais de eficiência. A análise econômica realizada pelo portal de dados europeu estima que as economias de custos acumuladas para a UE28+ em 2020 serão iguais a 1,7 mil milhões. EUR". ${ }^{4}$

Há, portanto, disposição para maior aproximação entre o Estado e o cidadão quando aquele abre seus dados, possibilitando a esse último a oportunidade de participar de maneira mais efetiva da governança pública com base na transparência, na confiança, na deliberação e no auxílio mútuo - sinais de uma democracia participativa.

Além da economia proporcionada ao Poder Público, existe um potencial econômico da abertura de dados, também para o setor privado, permitindo que pesquisadores e empreendedores possam desenvolver ferramentas tecnológicas que utiliza as informações disponibilizadas pelo Poder Público como parte do seu modelo de negócio. Veja-se, por exemplo, a possibilidade de o cruzamento de dados permitir o desenvolvimento de produtos e serviços dirigidos ao mercado de consumo, ao mercado empresarial e ao setor público, além de possibilitar a existência do incentivo ao surgimento de novas empresas e geração de empregos.

Nesse sentido, Attard et al. (2015, p. 339), esclarecem que os movimentos de abertura de dados surgiram, inicialmente, tendo como principais objetivos a transparência e o reuso de dados. De igual modo, a corrupção foi um fator determinante (mas não o único) para que estas iniciativas de disponibilização de dados abertos surgissem, uma vez que este desvio administrativo afeta não somente a economia pública, como também a própria sociedade.

\footnotetext{
${ }^{4}$ Tradução livre do autor (EUROPEAN DATA PORTAL).
} 
Os autores consideram, então, a transparência não como um fim, mas como um meio de desencorajar e combater a corrupção. Para eles, são três as razões principais para a abertura de dados governamentais: (1) transparência, porque é preciso monitorar as ações do Governo para garantir o funcionamento da democracia e a transparência permite, além do acesso aos dados, seu uso, reuso e distribuição, aumentando a possibilidade de controle social cidadão; (2) liberação de valor social e comercial, porque os dados produzidos pelo Governo em diferentes áreas têm um grande valor social e comercial e podem ser usados para propósitos diversos daqueles para os quais foram criados. Isso incentiva grupos interessados a criar novos serviços e produtos com estes dados; e (3) governança participativa, uma vez que, com a abertura de dados, os cidadãos têm a oportunidade de participar ativamente da Governança e do processo de tomada de decisões políticas, ao invés de apenas votarem esporadicamente em eleições.

Portanto, verifica-se que os benefícios do uso de dados abertos governamentais são diversos, estando diretamente associados a aspectos democráticos (como a participação; a colaboração; e a transparência) e impulsionadores da economia, especialmente no contexto da economia colaborativa. O potencial para gerar inovações tanto para o Estado quanto para a iniciativa privada demonstra a versatilidade no uso dos dados abertos governamentais.

\section{Estratégia de Dados Abertos da UFRN}

A UFRN é uma universidade de grande porte que procurou informatizar seus processos desde meados da década de 2000. Há uma forte cultura de progresso dentro da instituição e de informatização de seus serviços, com o intuito de facilitar o cotidiano de quem deles precisa e dos responsáveis em fornecê-los. Tendo em vistas as exigências legais e todos os benefícios expostos quanto à abertura de dados, a instituição resolveu estabelecer uma estratégia para liberação desses conjuntos de dados. Essa estratégia está relacionada ao longo histórico da instituição no desenvolvimento de sistemas eletrônicos para acompanhamento tanto da sua atividades-fim (ensino, pesquisa e extensão) quanto das atividades-meio (gestão, recursos humanos, patrimônio e processo).

Com a virtualização dos procedimentos institucionais viabilizada pelos sistemas de informação desenvolvidos na própria instituição, foi possível mapear os tipos de dados que já são cotidianamente inseridos no sistema pela comunidade universitária. Dessa maneira, garante-se maior eficiência e viabiliza-se a automação dos procedimentos de extração das bases de dados, que se torna independente da ação consciente dos atores institucionais que detêm a competência para gerar aqueles dados.

A primeira versão do PDA (Plano de Dados Abertos) foi elaborada em meados de 2016 por uma comissão nomeada pelo reitor em exercício da UFRN composta por representantes da Superintendência de Informática (Sinfo), Pró-reitoria de Planejamento e Ouvidoria. Além disso, o PDA foi devidamente apresentado e revisado por diversas unidades de gestão da instituição e, finalmente, aprovado pelo Conselho de Administração da UFRN em 29/09/2016. Para se estabelecer priorização dos conjuntos de dados disponibilizados, foram considerados o grau de relevância das informações para o cidadão, o alinhamento com os instrumentos de planejamento estratégico e a disponibilidades de dados já gerenciados pelos Sistemas Integrados de Gestão da UFRN (SIGs). 
Posteriormente, entre 27/10/2017 e 26/11/2017, foi realizada uma Hackathon dos dados abertos da UFRN. Naquela ocasião foram abertas novas bases de dados, permitindo que a comunidade acadêmica pudesse fazer uso delas no desenvolvimento de soluções relevantes para a instituição. O levantamento das bases de potencial interesse foi realizado em parceria pela Superintendência de Informática e pelo Laboratório de Governança Pública da UFRN, que resultou em um total de 58 conjuntos de dados, divididos em 11 grupos distintos.

Durante a revisão do PDA, um dos importantes atores na etapa de definição da priorização das bases a serem liberadas é a Ouvidoria. Partiu-se da premissa de que os pedidos de acesso à informação (LAI - Lei 12.527/11) realizados pelo canal e-SIC à Ouvidoria da UFRN seriam um bom indicador dos temas de maior interesse da população. Além disso, essa técnica de priorização possui como potencial efeito a diminuição da carga de trabalho da Ouvidoria e o aumento da eficiência na gestão, uma vez que os dados de maior interesse já seriam disponibilizados de modo ativo pela instituição, o que tende a diminuir o número de pedidos repetidos de acesso à informação.

Com base nessas premissas, utilizando-se os dados abertos do e-SIC (disponibilizados no portal de acesso à informação do Governo Federal), foi realizada uma análise dos pedidos de LAI direcionados à UFRN nos dois anos anteriores, o que é melhor visualizado através da figura abaixo:

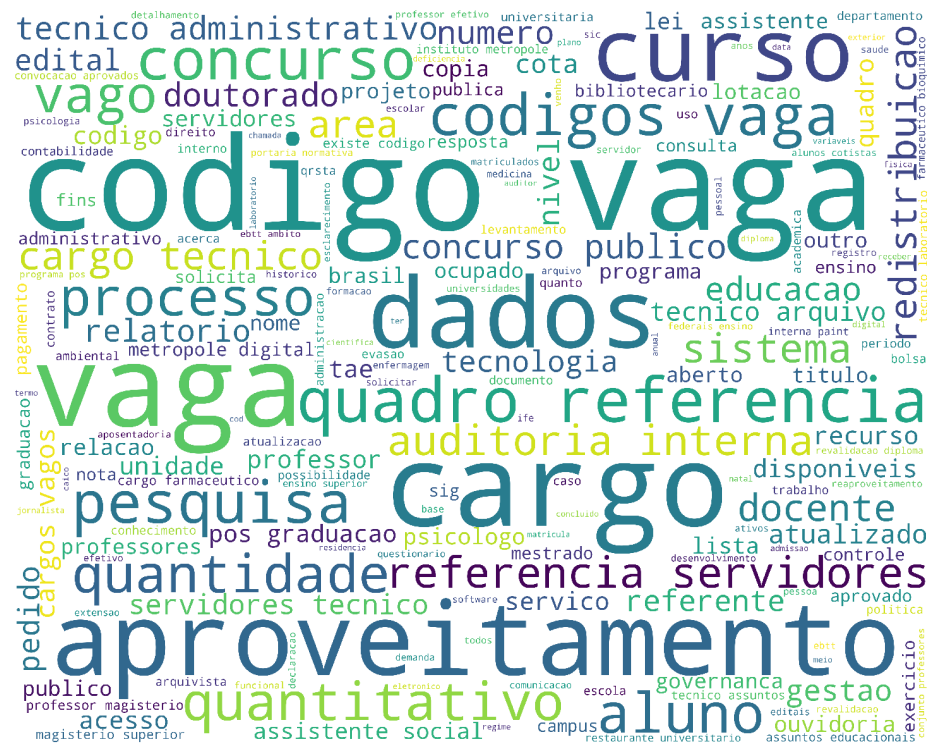

Figura 1. Nuvem das palavras mais comuns no campo "Resumo da Solicitação" nos pedidos de acesso à informação realizados através do canal e-SIC à Ouvidoria da UFRN entre 01/01/2016 e 25/08/2018. Fonte: elaboração própria com uso dos dados disponíveis no Portal de Dados Abertos do e-SIC.

A estratégia de dados abertos utilizada pela UFRN seguiu as sugestões de fases e tarefas do ProceDA (Processo para Dados Abertos), conforme definido na Seção 3.1. 


\subsection{ProceDA: um Processo para Dados Abertos em Instituições Públicas Brasileiras}

O ProceDA foi criado a partir de um amplo estudo da literatura sobre a disponibilização de dados abertos por instituições públicas. Esse estudo incluiu materiais de vários países, a partir dos quais foi possível obter uma lista de tarefas, boas práticas e fases para publicação de dados, que foram adaptados à realidade brasileira. O processo foi baseado no modelo de desenvolvimento de software Iterativo e Incremental (SILVA; JÚNIOR, 2018). A Figura 2 ilustra suas fases, bem como sua sequência.

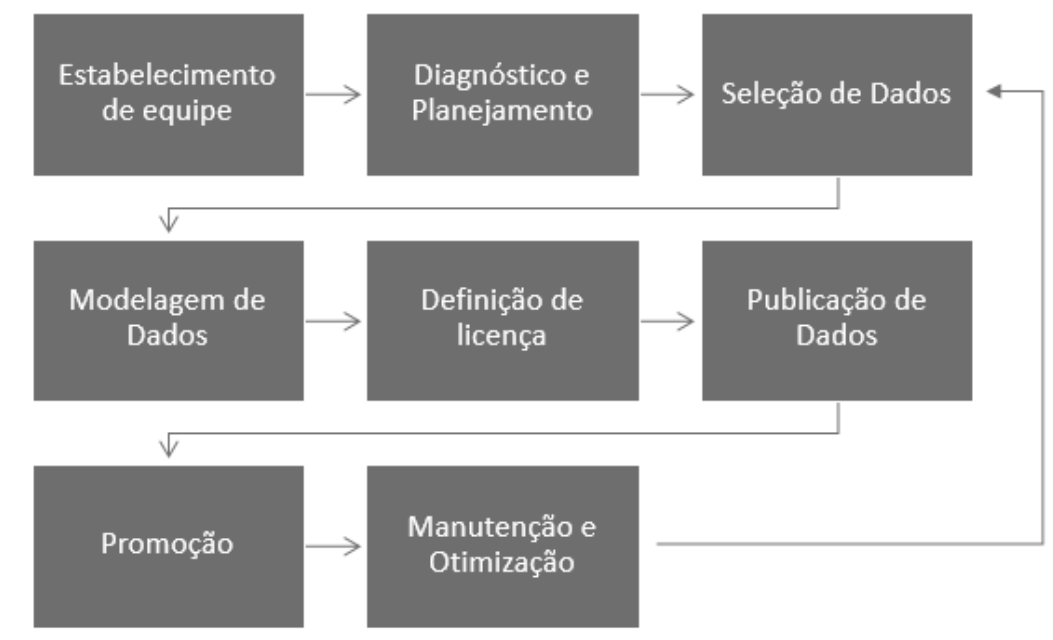

Figura 2. ProceDA e suas fases

As oito fases do processo são descritas a seguir:

- Estabelecimento de Equipe: estabelecimento de papéis e definição da equipe que irá atuar no projeto de dados abertos da instituição.

- Diagnóstico e Planejamento: avaliação geral da situação da instituição, através da medição de seu nível de preparo para disponibilização de dados e da realização de estudos de viabilidade do projeto; estabelecimento de um Plano de Dados Abertos; determinação de uma política de disponibilização de dados; estudo de legislação e outras questões gerais de projeto.

- Seleção de Dados: nesta etapa, ocorre a seleção dos conjuntos de dados que serão publicados no ciclo em questão. Deve-se observar uma série de questões para o estabelecimento de prioridades, como o valor associado aos conjuntos de dados, facilidade de publicação, benefícios e riscos da disponibilização de determinados dados, entre outros.

- Modelagem de Dados: nesta fase, ocorre a preparação dos dados para sua posterior publicação. A preparação inclui a limpeza desses dados, elaboração de metadados, tratamento de informações confidenciais e a escolha do melhor formato de publicação.

- Definição de Licença: deve-se definir os termos de uso dos dados, que devem ser, resumidamente, de formato aberto, não proprietários, estáveis e de amplo uso.

- Publicação de Dados: esta fase é composta por tarefas relacionadas à publicação, de fato, dos dados na Web.

- Promoção: são definidas estratégias de promoção dos dados que foram publicados, aumentando a chance de reuso pela comunidade. 
- Manutenção e Otimização: avaliação da necessidade de atualizar conjuntos de dados previamente publicados, além da realização de monitoramento e ajuste das atividades realizadas durante todo o processo, buscando encontrar possíveis pontos de melhoria.

O ProceDA conta com um total de cinco papéis. São eles:

- Órgão Central de Suporte e Controle das Ações (OCSCA): órgão que ficará responsável por auxiliar e coordenar o processo de abertura de dados, removendo eventuais obstáculos e problemas que surgirem durante o caminho, bem como articulando todas as unidades que irão abrir seus dados.

- Diretor: responsável pela iniciativa e principal interessado na abertura de dados. Geralmente é um Executivo da alta gestão, que pode ou não estar diretamente associado ao OCSCA.

- Coordenador: responde pela execução do processo de abertura e acompanhará de perto as tarefas executadas.

- Gestor Negocial: tem a capacidade de identificar dados importantes que podem ser abertos, os dados de risco e dados confidenciais.

- Gestor Técnico: responde pelas ações técnicas do processo de abertura, como extração, elaboração de conjuntos de dados e sua apresentação.

A Tabela 1 mostra todas as fases e tarefas do ProceDA, e como ocorreu a instanciação de cada uma delas no âmbito da UFRN.

Quanto à fase de Estabelecimento de Equipe, conforme destacado na Tabela, foram estabelecidos os seguintes responsáveis: Comitê Gestor de Tecnologia da Informação (CGTI), Coordenação do Serviço de Informação ao Cidadão, Pró-reitorias e unidades administrativas, e Superintendência de Informática. É possível fazer uma comparação entre esses papéis e os que foram propostos pelo ProceDA. Pode-se dizer que o CGTI exerceu, de forma simultânea, as funções do OCSCA, de Diretor e de Coordenador, pois esse comitê foi quem ficou responsável por auxiliar e coordenar todo o processo de abertura de dados, tendo realizado as tarefas referentes a esses três papéis. A Coordenação do Serviço de Informação ao Cidadão e as Pró-reitorias e unidades administrativas podem ser consideradas como equivalentes ao Gestor Negocial do ProceDA, tendo em vista que elas detêm o maior conhecimento sobre os dados a serem publicados e suas relevâncias. Por fim, a Superintendência de Informática exerceu o papel de Gestor Técnico, por deter os conhecimentos e pessoas necessárias à realização das tarefas da área. Portanto, conclui-se que houve correspondência total entre os papéis sugeridos pelo ProceDA e os que foram utilizados pela UFRN, apesar de alguns deles terem sido acumulados, algo que não é proibido pelo processo em questão.

Tabela 1. Lista de fases e tarefas do ProceDA e seu mapeamento com o que foi realizado pela UFRN

\begin{tabular}{|l|l|l|}
\hline Fase & Tarefa & Instanciação \\
\hline $\begin{array}{l}\text { Estabeleci- } \\
\text { mento de } \\
\text { Equipe }\end{array}$ & $\begin{array}{l}\text { Estabelecimento da equipe } \\
\text { responsável }\end{array}$ & $\begin{array}{l}\text { A equipe foi composta pelo CGTI (exerceu } \\
\text { papéis de OCSCA, Diretor e Coordenador), } \\
\text { Coordenação do Serviço de Informação ao } \\
\text { Cidadão (Gestor Negocial), Pró-reitorias e }\end{array}$ \\
\hline
\end{tabular}

iSys: Revista Brasileira de Sistemas de Informação (isys: Brazilian Journal of Information Systems) http://seer.unirio.br/index.php/isys/ 


\begin{tabular}{|c|c|c|}
\hline & & $\begin{array}{l}\text { unidades administrativas (Gestor Negocial) e } \\
\text { Superintendência de Informática (Gestor } \\
\text { Técnico). }\end{array}$ \\
\hline \multirow[t]{2}{*}{$\begin{array}{l}\text { Diagnóstico } \\
\text { e Planeja- } \\
\text { mento }\end{array}$} & $\begin{array}{l}\text { Realizar estudo de viabilidade } \\
\text { inicial }\end{array}$ & $\begin{array}{l}\text { A instituição considerou que possuía as } \\
\text { condições necessárias e não realizou essa tarefa } \\
\text { de maneira explícita, que envolvia, dentre } \\
\text { outras: a análise dos recursos materiais e } \\
\text { humanos disponíveis e do nível de preparo da } \\
\text { instituição, além de um estudo da legislação. }\end{array}$ \\
\hline & Estabelecer Plano de Dados Abertos & $\begin{array}{l}\text { O PDA da instituição foi construído e está } \\
\text { disponível ao público em seu portal oficials. }\end{array}$ \\
\hline \multirow[t]{2}{*}{$\begin{array}{l}\text { Seleção de } \\
\text { Dados }\end{array}$} & $\begin{array}{l}\text { Definir a estratégia de priorização } \\
\text { de conjuntos de dados }\end{array}$ & $\begin{array}{l}\text { Foram priorizados os dados mais solicitados por } \\
\text { meio do Serviço de Informação ao Cidadão, } \\
\text { dados já armazenados nos sistemas de } \\
\text { informação (SIGs), informações já publicadas } \\
\text { no Portal da Transparência da instituição e, por } \\
\text { fim, dados relevantes contidos nas páginas mais } \\
\text { acessadas nos portais da UFRN. Em resumo, } \\
\text { foram seguidas publicações orientadas a } \\
\text { demandas e a ofertas. }\end{array}$ \\
\hline & $\begin{array}{l}\text { Definir conjuntos de dados } \\
\text { prioritários e escolher os que serão } \\
\text { publicados }\end{array}$ & $\begin{array}{l}\text { Em cada ciclo de publicação, foram definidos os } \\
\text { conjuntos de dados de acordo com as estratégias } \\
\text { de priorização. }\end{array}$ \\
\hline \multirow[t]{6}{*}{$\begin{array}{l}\text { Modelagem } \\
\text { de Dados }\end{array}$} & $\begin{array}{l}\text { Modelar os dados em mais de um } \\
\text { formato }\end{array}$ & $\begin{array}{l}\text { Por questão de agilidade, os dados foram } \\
\text { publicados apenas em CSV. }\end{array}$ \\
\hline & $\begin{array}{l}\text { Manter um padrão nos formatos } \\
\text { publicados }\end{array}$ & $\begin{array}{l}\text { O formato de publicação é sempre o mesmo: } \\
\text { CSV. }\end{array}$ \\
\hline & $\begin{array}{l}\text { Definir nível de qualidade dos } \\
\text { dados }\end{array}$ & $\begin{array}{l}\text { Foram realizadas publicações no nível de } 3 \\
\text { estrelas, de acordo com a classificação de } \\
\text { Berners-Lee (2006). }\end{array}$ \\
\hline & Elaborar metadados & Metadados são sempre elaborados. \\
\hline & Limpeza dos dados & $\begin{array}{l}\text { Os responsáveis pelos dados devem verificá-los, } \\
\text { homologando-os antes de serem publicados. }\end{array}$ \\
\hline & Tratar informações confidenciais & Informações confidenciais ou sensíveis não são \\
\hline
\end{tabular}




\begin{tabular}{|c|c|c|}
\hline & & publicadas. \\
\hline $\begin{array}{l}\text { Definição de } \\
\text { Licença }\end{array}$ & $\begin{array}{l}\text { Especificar uma licença para os } \\
\text { dados }\end{array}$ & Foi escolhida a Licença Aberta, sem restrições. \\
\hline \multirow[t]{5}{*}{$\begin{array}{l}\text { Publicação } \\
\text { de Dados }\end{array}$} & $\begin{array}{l}\text { Publicar os dados no portal de } \\
\text { catalogação }\end{array}$ & $\begin{array}{l}\text { Os dados encontram-se publicados no portal de } \\
\text { catalogação oficial da instituição. }\end{array}$ \\
\hline & $\begin{array}{l}\text { Informar a versão dos conjuntos de } \\
\text { dados }\end{array}$ & $\begin{array}{l}\text { O número da versão e a última data de } \\
\text { atualização são exibidos nas páginas de cada } \\
\text { conjunto de dados. }\end{array}$ \\
\hline & Publicar metadados & $\begin{array}{l}\text { Foram publicados para todos os conjuntos de } \\
\text { dados. }\end{array}$ \\
\hline & $\begin{array}{l}\text { Elaborar URLs únicas e persistentes } \\
\text { para os conjuntos de dados }\end{array}$ & $\begin{array}{l}\text { As URLs de cada conjunto de dados são únicas, } \\
\text { não se alteram com o tempo e possuem um } \\
\text { padrão de formação. }\end{array}$ \\
\hline & $\begin{array}{l}\text { Explicitar termos de uso no portal } \\
\text { de catalogação }\end{array}$ & $\begin{array}{l}\text { Na página de cada conjunto de dados, é exibida } \\
\text { sua respectiva licença. }\end{array}$ \\
\hline \multirow[t]{3}{*}{ Promoção } & $\begin{array}{l}\text { Publicar em redes sociais e sites } \\
\text { oficiais sobre os conjuntos de dados }\end{array}$ & $\begin{array}{l}\text { Foram publicadas notícias quando o portal de } \\
\text { catalogação foi disponibilizado e para promoção } \\
\text { do I hackathon da instituição. }\end{array}$ \\
\hline & Considerar eventos maiores & Foi realizado um hackathon. \\
\hline & $\begin{array}{l}\text { Integrar catálogo da instituição ao } \\
\text { catálogo central do governo }\end{array}$ & A integração foi realizada. \\
\hline \multirow{5}{*}{$\begin{array}{l}\text { Manuten- } \\
\text { ção e } \\
\text { Otimização }\end{array}$} & $\begin{array}{l}\text { Verificar se algum conjunto de } \\
\text { dados precisa ser atualizado }\end{array}$ & $\begin{array}{l}\text { Cada conjunto de dados possui seu tempo de } \\
\text { atualização pré-definido. }\end{array}$ \\
\hline & $\begin{array}{l}\text { Monitoramento e ajuste da execução } \\
\text { do processo }\end{array}$ & Está previsto no PDA e sendo executada. \\
\hline & $\begin{array}{l}\text { Verificar como os dados estão } \\
\text { sendo usados pela comunidade }\end{array}$ & $\begin{array}{l}\text { Periodicamente são coletados dados de acesso } \\
\text { aos conjuntos de dados abertos. }\end{array}$ \\
\hline & $\begin{array}{l}\text { Facilitar requisições de dados } \\
\text { através de portais oficiais }\end{array}$ & É possível realizar requisições através do e-SIC. \\
\hline & Facilitar feedback dos usuários & Os feedbacks são enviados através de um email \\
\hline
\end{tabular}


\begin{tabular}{|l|l|l|}
\hline & $\begin{array}{l}\text { sobre conjuntos de dados } \\
\text { individuais }\end{array}$ & específico divulgado no portal de dados abertos. \\
\hline
\end{tabular}

Conforme é possível ver na Tabela 1, no geral, houve uma boa correspondência entre o que foi previsto pelo ProceDA e o que foi executado pela instituição. Não foram identificados maiores problemas ou desafios associados à utilização desse processo.

Apesar de a UFRN ter sido bem-sucedida na publicação de seus dados, algumas dificuldades foram enfrentadas, tais como: diversidade na quantidade de dados e atores envolvidos; conseguir articular a equipe para que os dados relevantes fossem de fato publicados; fazer os envolvidos compreenderem o que são dados abertos e quais seus objetivos. No entanto, avaliou-se que essas questões poderiam ter sido amenizadas caso o estudo de viabilidade inicial, juntamente à sugestão de lidar com o pessoal envolvido, removendo dúvidas e obstáculos, tivessem sido realizados.

\subsection{Infraestrutura tecnológica}

Os sistemas de gestão desenvolvidos na UFRN são atores fundamentais no processo de abertura dos dados da Instituição, visto que geram grandes quantidades de dados sobre suas diversas áreas. Particularmente, existem informações relativas às atividades de ensino, pesquisa, extensão, pessoas, patrimônio, despesas, orçamento, entre outros. Esses conteúdos são essenciais para a promoção da transparência e propiciam a geração de inovações que possam trazer benefícios para a sua própria gestão, assim como para a comunidade acadêmica.

O Portal de Dados Abertos da UFRN é o sistema responsável por catalogar e disponibilizar, para toda a população, dados públicos gerados pelos SIGs e de outros meios de geração de dados da Instituição, possibilitando a concretização do acesso à informação (regulamentado pela Lei de Acesso à Informação Pública - Lei $\mathrm{n}^{\mathrm{o}}$ 12.527/2011) e o acompanhamento do funcionamento da instituição.

Esse portal foi implantado utilizando a plataforma de código aberto CKAN (Comprehensive Knowledge Archive Network). Sua escolha se deu principalmente pela expressiva adoção por outros órgãos nacionais e internacionais, inclusive pelo próprio governo brasileiro - o que facilitou a integração entre os dois portais. A ferramenta possui uma intuitiva interface Web, a qual facilita o uso por quaisquer potenciais usuários, e é composta por um mecanismo robusto de buscas, meios de organização dos dados em entidades (organizações, grupos, conjuntos de dados e recursos), painéis de exibição de metadados relacionados a essas entidades (datas de atualização e criação, títulos, descrições, versões, licenças e outros) e mecanismos de visualização dos dados (gráficos, tabelas, mapas etc.) (CKAN, 2019).

O CKAN permite estruturar os conjuntos de dados (datasets) entre duas entidades, sendo elas os grupos e organizações, podendo o dataset estar em vários grupos e/ou em uma organização específica, e podendo possuir um ou mais recursos em formato de texto (como CSV, JSON, XML, PDF, TXT e outros). Um órgão pode ter vários dos seus departamentos como organizações que se auto gerenciam de forma independente (CKAN, 2019). No caso da UFRN, a SINFO foi designada como o órgão responsável por gerenciar todos os dados catalogados no portal. Por isso, há uma única 
organização com diferentes grupos que agregam os conjuntos de dados em escopos mais bem definidos (por exemplo, Comunicados e Documentos, Contratos e Convênios, Materiais, Processos etc.). Já no portal do Governo, existem centenas de organizações. Cada uma destas gerencia seus próprios datasets, que podem estar reunidos entre os grupos do próprio Portal.

Além disso, o CKAN possui uma rica API (Application Programming Interface) - muito bem documentada - que possibilita o consumo de seus dados e metadados através de aplicações terceiras (CKAN, 2019). A API do CKAN propicia também atualizações automáticas dos dados catalogados, o que facilitou a criação de um sistema para gerenciar as atualizações periódicas de dados no portal, o SIDA (Sistema Integrado de Dados Abertos). O SIDA possui scripts de bancos de dados para cada um dos recursos dos datasets do portal e faz as atualizações desses recursos em períodos definidos pelo usuário mantenedor desse sistema. Dessa forma, garante-se que o portal de dados da UFRN se mantenha, com pouca interferência manual, sempre atualizado.

\section{Resultados obtidos}

O rápido crescimento da oferta de conjuntos de dados sobre pessoas, artefatos tecnológicos e organizações nos levou a uma posição em que temos à disposição grandes quantidades de informações prontas para serem rearranjadas e modeladas com $o$ objetivo de criar valor adicional. Dados abertos estruturados e processáveis por máquinas, que são livres para acessar e interligar, estão se tornando o futuro para o desenvolvimento de inovações em ambientes institucionais (NAJDENOV et al., 2015). Apesar de já existirem inúmeros exemplos de como os dados abertos estão criando valor tanto social quanto econômico, ainda não é possível afirmar quais novos recursos, tecnologias ou serviços se tornarão disponíveis (INTERNATIONAL, 2018).

Neste sentido, esta seção tem como objetivo demonstrar de forma concreta como a iniciativa de dados abertos da UFRN, alinhada com políticas de suporte e fomento ao consumo desses dados, possibilitou inúmeros benefícios para a própria instituição. De fato, estes resultados não seriam possíveis sem uma iniciativa como esta. Além do mais, foi notável como, em pouco tempo após a publicação dos dados, começaram a surgir iniciativas relevantes por parte da comunidade acadêmica.

\subsection{Dados Aberto como impulsionador da transparência}

Desde a divulgação do Portal de Dados da UFRN até o momento atual, houve 192.084 acessos às suas páginas, realizados por mais de 15.000 usuários. Desse total de acessos, 37.009 foram feitos aos 58 datasets presentes no Portal. Os datasets mais acessados (entre 1000 e 2943 acessos) são os que possuem informações de discentes, cursos, turmas, matrículas em componentes, docentes, servidores e gastos. Esses e outros dados catalogados no Portal são frequentemente utilizados em atividades de disciplinas e trabalhos de fim de cursos dos níveis de graduação e de pós-graduação da Instituição, como também em seus projetos de ensino, pesquisa e extensão.

Iniciativas como essas são valiosas para ajudar a instituição na tomada de decisões, podendo trazer benefícios para a comunidade acadêmica, além de ganhos para a própria gestão. Isso é possível quando os dados disponibilizados possuem alto valor agregado, possibilitando à própria sociedade explorá-los de maneira mais efetiva do que os próprios donos daqueles dados, que geralmente possuem apenas uma visão limitada

iSys: Revista Brasileira de Sistemas de Informação (isys: Brazilian Journal of Information Systems) http://seer.unirio.br/index.php/isys/ 
sobre eles, utilizando-os para atividades básicas (JANSSEN; CHARALABIDIS; ZUIDERWIJK, 2012). Além disso, os dados abertos da instituição juntamente com essas iniciativas formam um mecanismo de prestação de contas para a população, mostrando o uso dos recursos públicos não só por parte da Instituição, mas também dos seus servidores e alunos. A seguir serão demonstrados alguns exemplos dessas iniciativas.

\subsubsection{Monografias de Discentes da Instituição}

Dentre os possíveis campos de estudos que podem ser feitos com dados contidos no Portal, percebe-se uma tendência voltada para trabalhos que estudam as influências da evasão e desempenho dos discentes da universidade, analisando dados socioeconômicos e histórico do aluno. Com essa temática, uma aluna do curso de graduação em Engenharia de Computação da UFRN desenvolveu seu trabalho de conclusão de curso. O trabalho teve como objetivo traçar o perfil dos alunos antes de entrar na universidade até o momento da evasão nos seus respectivos cursos.

Outro trabalho, intitulado como "Análise de Dados de Ensino, Pesquisa e Extensão do Portal de Dados Abertos da UFRN", foi desenvolvido por um aluno do curso de Especialização em Big Data da universidade. $\mathrm{O}$ estudo teve como objetivo a validação dos dados disponíveis no Portal e a avaliação da viabilidade da análise desses recursos como forma de prover informações relevantes sobre Ensino, Pesquisa e Extensão na UFRN, com intuito de auxiliar a gestão universitária.

\subsubsection{Laboratório de Governança Pública da UFRN}

A disponibilização de dados em formato aberto vem sendo utilizada também como ferramenta para desenvolvimento das atividades-fim da instituição. Exemplos disso são os projetos desenvolvidos pelo Laboratório de Governança Pública da UFRN, cujo tema central é analisar a existência de possível correlação entre a maior disponibilização de conjuntos de dados abertos e a melhoria na capacidade de identificação e gerenciamento de riscos (YOUNG e VERHULST, 2016) com foco na análise da UFRN a partir da análise de outros casos na administração pública federal (BEGHIN e ZIGONI, 2014).

\subsection{Acesso a dados como impulsionador da inovação}

Ter vencido a barreira inicial de planejar, implantar e sustentar uma política efetiva de dados abertos nos proporcionou uma oportunidade para desenvolver uma ação ainda mais desafiadora e complementar. Particularmente, com o objetivo de descentralizar o esforço de inovação em soluções de TI, impulsionando um ambiente de empoderamento do usuário e, acima de tudo, de promoção da cultura de inovação sem permissão (THIERER, 2014).

O passo inicial para isto foi a construção e publicação de um rico conjunto de APIs baseadas em uma arquitetura moderna e acessível a toda comunidade acadêmica. Vale salientar que a solução da API extrapola a solução de dados abertos, no sentido de que ela fornece não só acesso aos dados públicos, mas também aos dados privados dos usuários. Neste caso, é necessário que o usuário (dono da informação) conceda explicitamente o acesso às suas informações ao sistema ou aplicativo de terceiros. Esta concessão se baseia no mesmo modelo de segurança amplamente utilizado por diversas soluções, como o Facebook, Google e Twitter. A fim de manter a solução sustentável e 
cumprir seu propósito original, foi fundamental estabelecer um conjunto de políticas destinadas a: (a) incentivar o desenvolvimento de soluções conectadas à API; (b) Manter a confiabilidade na solução por meio de suporte técnico adequado e agilidade na solução de problemas; C) fornecer segurança e apoio legal aos desenvolvedores sobre a garantia de direitos autorais das inovações desenvolvidas.

Apesar da importância da tecnologia e da política, o ponto chave da estratégia foi a adoção dos princípios do modelo de inovação sem permissão. Particularmente, a ideia que todos da comunidade acadêmica (sem exceção) estariam livres para experimentar e criar a solução que desejassem sem ter que pedir permissão para nenhuma unidade ou gestor da instituição foi $o$ grande incentivador para $o$ desenvolvimento descentralizado de inovações. O uso mais comum é pelos discentes para desenvolvimento de projetos em disciplinas, muitas vezes incentivados pelos próprios docentes. Outro exemplo muito comum é o de professores no desenvolvimento de seus projetos de pesquisas e estudos com seus orientandos. Servidores das unidades acadêmicas e administrativas, com conhecimentos técnicos em TI, também fazem uso da API para desenvolver soluções específicas para suas unidades. Além desses, outros usos também foram observados, tais como: em hackathons, em soluções de empresas incubadas e em trabalhos de conclusão de curso de graduação e pós-graduação. Enfim, todas estas iniciativas naturalmente emergindo de forma descentralizada em um ambiente acadêmico, que por natureza é um local fértil para a produção de conhecimento, geraram o que podemos considerar como um ecossistema de inovação.

Como resultado desta iniciativa pudemos observar a criação de mais de 50 soluções de software desenvolvidos por membros da comunidade acadêmica da UFRN, envolvendo aplicativos para celular e sistemas Web, que utilizam os dados abertos como subsídio para suas funcionalidades. Esses dados são providos através do mecanismo de API, que oferece acesso aos dados reais dos sistemas institucionais. Por questões de segurança e desempenho, a API usa um banco de dados independente. No entanto, qualquer dado alterado nos sistemas é sincronizado imediatamente para o banco de dados utilizado pela API.

Atualmente, aproximadamente 40 aplicações que fazem uso da API estão em ambiente de produção e geram uma média semanal de 53.000 acessos às operações da API (com picos de 139.000 em certas semanas). Isso demonstra o potencial das inovações disruptivas, produzidas de forma descentralizada pelos próprios usuários, induzidas pela criação da solução API e pelo suporte de uma política de inovação sem permissão. Dentre as 40 aplicações que hoje usam a API, que estão espalhadas nos mais diversos segmentos, existem aplicações que focam na segurança, mais especificamente no controle das viaturas na instituição; aplicação que realiza a gestão energética da sala, apagando a luz e o ar-condicionado logo depois que não encontrar mais nenhum movimento por um determinando período de tempo, desligando as luzes e o aparelho de ar ao término da última aula.

Serão demonstradas a seguir, em maiores detalhes, a relevância dos resultados de três iniciativas conectadas à API.

\subsubsection{Ferramenta Multiprova}

A ferramenta Multiprova é um sistema desenvolvido pelo esforço coletivo de professores ligados a um departamento da área de exatas, que buscou formas de 
melhorar o processo de ensino/aprendizagem, principalmente no contexto de aulas de calouros com mais de 100 alunos por turma. Para isto, eles construíram uma ferramenta destinada a simplificar e automatizar as etapas operacionais de organizar, corrigir, analisar e compartilhar informações sobre exames. Mais especificamente, o Multiprova permite que os professores gerem, de forma controlada, variações nos aspectos acessórios dos enunciados e elementos alternativos nas questões. A partir de uma única pergunta básica, você pode gerar variações em itens como números, palavras, fórmulas, gráficos e imagens.

Para essa ferramenta funcionar de forma adequada, foi necessário usar os serviços da API para acessar os dados acadêmicos oficiais, como disciplinas (programas, referências, etc.), docentes e estudantes. Atualmente, o Multiprova é amplamente utilizado na universidade. Em apenas dois anos de operação, 3.200 perguntas foram criadas e mais de 800 avaliações foram aplicadas a aproximadamente 20.000 alunos.

\subsubsection{VemCar - Um aplicativo de caronas}

O problema da mobilidade urbana é um desafio enfrentado pela maioria das grandes cidades do mundo. O hábito de usar o próprio carro em vez de transporte público é uma das causas desse problema. Considerando essa situação, um aluno, em um projeto de disciplina, desenvolveu uma solução para promover o compartilhamento de veículos, facilitando um sistema de compartilhamento de caronas. O aplicativo VemCar, atualmente publicado na Play Store, é restrito a membros da comunidade acadêmica da UFRN e permite que sejam oferecidas e solicitadas caronas no caminho para ou a partir dos campi universitários de maneira prática, rápida e segura.

Apesar da existência de outras aplicações para o mesmo fim, essa solução é mais adequada ao contexto da universidade, pois integra-se à API, restringindo o acesso apenas aos usuários oficialmente vinculados à instituição. A solução aborda a preocupação de mobilidade urbana e sustentabilidade, ajudando a preservar a segurança dos passageiros.

\subsubsection{Reuse - Sistema para reuso de bens}

O aplicativo Reuse foi concebido em uma pesquisa de mestrado e visa a melhorar o uso de bens duráveis da universidade, estimulando e facilitando a troca e transferência de itens patrimoniais entre as unidades da UFRN. A ferramenta aborda esse problema fornecendo funcionalidades para a ampla distribuição dos bens e facilitando o processo de transferência para a parte interessada. Suas características e usabilidade foram inspiradas em sistemas conhecidos de negociação, venda e compra de itens como OLX, Mercado Livre e Etsy. O reuse está conectado à API e, portanto, tem acesso a dados oficiais relacionados a unidades, bens e usuários.

\section{Oportunidades e Considerações Finais}

Pelo exposto, é visível a conexão entre o regime democrático e a abertura de dados pelo Poder Público. Do ponto de vista teórico, objetivou-se demonstrar a relação entre dados abertos governamentais e o aumento da eficiência na administração pública como um todo, o que viabiliza uma maior transparência e concretização dos gastos públicos. 
Neste trabalho, demonstrou-se que para que essa abertura ocorra é necessária uma concatenação de fatores, desde a obrigação legal de publicar dados, passando pela existência de uma estratégia de abertura bem delimitada na gestão, até a definição de um processo com responsabilidades definidas. No caso estudado, a disponibilidade prévia dos SIGs foi essencial para o sucesso da política institucional de dados abertos, uma vez que geram grande quantidade de dados relacionados às atividades finalísticas e meio da instituição. Além disso, um ponto de destaque foi a visão estratégica de extrapolar a exigência legal e realmente oferecer um grande e rico portfólio de dados abertos, além da API para os sistemas. Como efeito em cascata, atividades ligadas ao ensino, à pesquisa e à extensão foram fortalecidas com a liberação das bases de dados em formato aberto. Vários exemplos de usos destes dados em estudos, pesquisas e trabalhos de conclusão de curso puderam ser observados. Adicionalmente, é relevante notar que a estratégia de abertura adotada também teve um papel impulsionador da inovação tecnológica, o que foi possível perceber a partir dos exemplos de soluções desenvolvidas de forma descentralizada e que consomem dados da instituição.

Para uma ampliação dos benefícios produzidos pelas iniciativas de dados abertas no Brasil, torna-se imprescindível maior engajamento popular para que o PL 7804/2014 se torne Lei. O objetivo principal do PL 7804/2014 é criar a obrigatoriedade para a disponibilização de dados abertos e de interfaces de aplicações Web de forma organizada e estruturada para a União, estados e municípios. O ecossistema de dados abertos no Brasil só estará plenamente estabelecido quando todos os poderes, em todas as esferas, forem obrigados a divulgar os seus dados em formato aberto. As possibilidades para a melhoria da sociedade brasileira serão inúmeras, afinal, a história dos regimes democráticos mostra que a solução para os problemas que assolam as democracias é, sempre, mais democracia.

\subsection{Contribuições do Trabalho}

Entre as contribuições deste trabalho, destacam-se:

- discussão sobre dados abertos e seus benefícios;

- apresentação de um processo para abertura de dados e explicação de como ele foi e pode ser utilizado;

- incentivo à transparência pública, simultaneamente ao desenvolvimento da inovação;

- relato das lições aprendidas na aplicação de uma abordagem sistemática de abertura de dados;

- apresentação de boas práticas no processo de publicação dos conjuntos de dados, através de automatização, e exposição de ferramentas e características para alavancar o Portal de Dados Abertos de uma instituição.

\subsection{Limitações e Trabalhos Futuros}

Este artigo apresentou a estratégia de abertura de dados especificamente adotada pela UFRN. De certa forma, pode-se considerar que as condições e características específicas da instituição no momento da implantação foram os fatores determinantes do seu sucesso. Certamente, não podemos afirmar que a estratégia aqui descrita oferece uma garantia de obtenção dos mesmos resultados. No entanto, as práticas adotadas e os princípios por trás do ProceDA representam uma compilação sinérgica das melhores 
práticas envolvendo iniciativas de dados abertos governamentais. Desta forma, acreditamos que outras instituições de natureza similar possam também obter resultados positivos com adoção desta abordagem.

Do ponto de vista mais geral, a atual exigência legal para abertura de dados é de fato bastante restrita, uma vez que o Decreto 8.777/16 atinge apenas o Poder Executivo Federal. Tais restrições dificultam a formação de um ecossistema amplo de dados abertos, com muito mais potencial para gerar pesquisa e inovação. A ausência do dever de abertura para os demais Poderes (Legislativo e Judiciário) e esferas (estadual e municipal) demonstra que o Brasil ainda sofre com um relevante déficit democrático, com impacto negativo direto na transparência e controle social. Desta forma., consideramos imprescindível a condução de iniciativas tais como a PL 7804/2014, que atualmente tramita na Câmara dos Deputados.

\section{Agradecimentos}

Os autores agradecem às agências de fomento CAPES e CNPq pelos financiamentos que viabilizaram a realização de eventos sobre dados abertos governamentais. Agradecem, ainda, o apoio institucional da alta gestão da UFRN na adoção de medidas que ultrapassam o mero cumprimento da política de dados abertos do Governo Federal.

\section{Referências}

ANDERSEN, T. B. (2009). E-Government as an anti-corruption strategy. Information Economics and Policy, 21(3), 201-210. doi: http://dx.doi.org/10.1016/j.infoecopol.2008.11.003

ATTARD, J.; ORLANDI, F.; SCERRI, S.; AUER, S. A systematic review of open government data initiatives. Government Information Quarterly, [s.l], [s.n.], v. 32, 2015, p. 399-418. [GS Search]

BARGH, M. S.; CHOENNI, S.; MEIJER, R. Meeting open data halfway: On semi-open data paradigm. In: Proceedings of the 9th International Conference on Theory and Practice of Electronic Governance. [S.1.]: Association for Computing Machinery, 2016. p. 199-206. [GS Search]

BEGHIN, N.; ZIGONI, C. Avaliando os websites de transparência orçamentária nacionais e sub-nacionais e medindo impactos de dados abertos sobre direitos humanos no Brasil. Brasília: Instituto de Estudos Socioeconômicos, 2014. 117 p. ISBN 978-85-87386-31-1. [GS Search]

BERNERS-LEE, T. Linked Data. Jul., 2006. Disponível em: <https://www.w3.org/DesignIssues/LinkedData.html>. Acesso em 19 Nov., 2018.

BERTOT, J. C.; JAEGER, P. T.; GRIMES, J. M. (2010). Using ICTs to create a culture of transparency: E-government and social media as openness and anti-corruption tools for societies. Government Information Quarterly, 27(3), 264-271. doi: http://dx.doi.org/10.1016/j.giq.2010.03.001 
BOBBIO, N. Estado, governo, sociedade: para uma teoria geral da política. Tradução de Marco Aurélio Nogueira. 14. ed. Rio de Janeiro: Paz e Terra, 2017. [GS Search]

BOBBIO, N. O futuro da democracia. Tradução de Marco Aurélio Nogueira. 6. ed. Rio de Janeiro: Paz e Terra, 2015. [GS Search]

BRASIL. Decreto ${ }^{\circ}$ 8777, de 11 de maio de 2016. Institui A Política de Dados Abertos do Poder Executivo Federal. Brasília, 11 maio 2016. Disponível em: <http://www.planalto.gov.br/ccivil 03/_ato2015-2018/2016/decreto/D8777.htm>. Acesso em 29 Nov., 2018.

BRASIL. O que são dados abertos? Brasília, 2018. Disponível em: < http://dados.gov.br/pagina/dados-abertos>. Acesso em 29 Nov., 2018.

CHAN, C. "From open data to open innovation strategies: Creating e-services using open government data." 2013 46th Hawaii International Conference on System Sciences. IEEE, 2013. [GS Search]

CKAN. CKAN Features. Disponível em: <https://ckan.org/features/>. Acesso em: 03 abr. 2019.

EUROPEN DATA PORTAL. Open Data in a nutshell. Disponível em: $<$ https://www.europeandataportal.eu/en/providing-data/goldbook/open-datanutshell>. Acesso em 29 nov., 2018.

GOVERNO DO BRASIL. Brasil é o oitavo país no ranking mundial de dados abertos. Disponível em: <http://www.brasil.gov.br/governo/2017/04/brasil-e-o-oitavo-paisno-ranking-mundial-de-dados-abertos>. Acesso em: 29 nov. 2018.

INTERNATIONAL FEDERATION OF ACCOUNTANTS. International Framework: good governance in the public sector. New York: International Federation of Accountants, $2014 . \quad 42$ p. 42 Disponível em: $<$ https://www.cipfa.org/ /media/files/publications/standards/ifac/internationalframew orkgoodgovernanceinthepublicsectorifaccipfa2.pdf>. Acesso em 29 nov., 2018

INTERNATIONAL, O. K. Open Data Handbook. out. 2017. Disponível em: $<$ http://opendatahandbook.org>. Acesso em 20 Nov., 2018.

JANSSEN, M.; CHARALABIDIS, Y.; ZUIDERWIJK, A. Benefits, adoption barriers and myths of open data and open government. Information Systems Management (ISM), v. 29, n. 4, p. 258-268, 2012. [GS Search]

KUCERA, J. et al. Methodologies and best practices for open data publication. In: Proceedings of the Dateso 215. [S.1.]: CEUR-WS, 2016. p. 52-64. [GS Search]

NAJDENOV, B. et al. Open financial data from the macedonian stock exchange. In: Proceedings of the 6th Information and Communication Technologies Innovations 2014 conference. [S.1.]: Springer Verlag, 2015. p. 115-124. [GS Search] 
OPEN KNOWLEDGE FOUNDATION. Definição de conhecimento aberto. Disponível em <http://opendefinition.org/od/2.0/pt-br/>. Acesso em 29 nov., 2018.

PARKS, W.. Open Government Principle: Applying the Right to Know Under the Constitution. The George Wawhington Law Review, 1957, 26(1). [GS Search]

PICAZO-VELA, S.; FERNANDEZ-HADDAD, M.; LUNA-REYES, L. F. IT's alive!!: social media to promote public health. Proceedings of the 14th Annual International Conference on Digital Government Research. Quebec, 2013, Canada: ACM. doi:10.1145/2479724.2479743. [GS Search]

PORTAL BRASILEIRO DE DADOS ABERTOS. O que são dados abertos? Disponível em: <http://dados.gov.br/pagina/dados-abertos>. Acesso em: 29 nov. 2018.

RUIJER, E.; GRIMMELIKHUIJSEN, S.; MEIJER, A. Open data for democracy: developing a theoretical framework for open data use. Government Information Quarterly, [s.1.], [s.n.], v. 34, Issue 1, 2017, p. 46. [GS Search]

SILVA, R. O.; AQUINO JÚNIOR, G. S. Uma Proposta de Processo para Implantação de dados Abertos em Instituições Públicas Brasileiras. iSys I Revista Brasileira de Sistemas de Informação, Rio de Janeiro, vol. 11, No. 1, pp. 30-54, 2018.

THIERER, A. Permissionless Innovation: The Continuing Case for Comprehensive Technological Freedom. Mercatus Center at George Mason University, 2014. [GS Search]

TRIBUNAL DE CONTAS DA UNIÃO. Governança Pública: referencial básico de governança aplicável a órgãos e entidades da administração pública e ações indutoras de melhoria. Brasília: Tribunal de Contas da União, 2014. 96 p. Disponível em: $<$ http://www.fazenda.gov.br/pmimf/institucional/download-de-arquivos/governancapublica-tcu.pdf>. Acesso em 29 nov., 2018.

VIEIRA, F. M.; SANTOS, V. V. B. D. Governo eletrônico: a busca por um governo mais transparente e democrático. 2010. [GS Search]

YOUNG, A.; VERHULST, S. The Global Impact of Open Data. Sebastopol: O'Reilly, 2016. ISBN 978-1-491-96467-5. Disponível em $<$ https://data.gov.ru/sites/default/files/documents/the-global-impact-of-open-data.pdf >. Acesso em 29 nov., 2018.

ZUIDERWIJK, A., JANSSEN, M. AND DAVIS, C., 2014. Innovation with open data: Essential elements of open data ecosystems. Information Polity, 19(1, 2), pp.17-33. [GS Search] 